\title{
THE ANGLO-AUSTRALIAN PLANET SEARCH. XXII. TWO NEW MULTI-PLANET SYSTEMS
}

\author{
Robert A. Wittenmyer ${ }^{1}$, J. Horner ${ }^{1}$, Mikko Tuomi ${ }^{2,3}$, G. S. Salter ${ }^{1}$, C. G. Tinney ${ }^{1}$, R. P. Butler ${ }^{4}$, H. R. A. Jones ${ }^{5}$, \\ S. J. O'Toole ${ }^{6}$, J. Bailey ${ }^{1}$, B. D. Carter ${ }^{7}$, J. S. Jenkins ${ }^{8}$, Z. Zhang ${ }^{2}$, S. S. Vogt ${ }^{9}$, And Eugenio J. Rivera ${ }^{9}$ \\ ${ }^{1}$ Department of Astrophysics, School of Physics, University of New South Wales, Sydney, NSW 2052, Australia; rob@ phys.unsw.edu.au \\ ${ }^{2}$ Centre for Astrophysics Research, Science and Technology Research Institute, University of Hertfordshire, College Lane, Hatfield AL10 9AB, UK \\ ${ }^{3}$ Department of Physics and Astronomy, University of Turku, Tuorla Observatory, Väisäläntie 20, FI-21500 Piikkiö, Finland \\ ${ }^{4}$ Department of Terrestrial Magnetism, Carnegie Institution of Washington, 5241 Broad Branch Road, NW, Washington, DC 20015-1305, USA \\ ${ }^{5}$ Centre for Astrophysics Research, University of Hertfordshire, College Lane, Hatfield, AL10 9AB, UK \\ ${ }^{6}$ Australian Astronomical Observatory, P.O. Box 296, Epping, NSW 1710, Australia \\ ${ }^{7}$ Faculty of Sciences, University of Southern Queensland, Toowoomba, Queensland 4350, Australia \\ ${ }^{8}$ Departamento de Astronomía, Universidad de Chile, Camino El Observatorio 1515, Las Condes, Santiago, Chile \\ ${ }^{9}$ UCO/Lick Observatory, University of California, Santa Cruz, CA 95064, USA \\ Received 2012 February 19; accepted 2012 May 11; published 2012 June 26
}

\begin{abstract}
We report the detection of two new planets from the Anglo-Australian Planet Search. These planets orbit two stars each previously known to host one planet. The new planet orbiting HD 142 has a period of $6005 \pm 427$ days, and a minimum mass of $5.3 M_{\text {Jup }}$. HD $142 \mathrm{c}$ is thus a new Jupiter analog: a gas-giant planet with a long period and low eccentricity $(e=0.21 \pm 0.07)$. The second planet in the HD 159868 system has a period of $352.3 \pm 1.3$ days and $m \sin i=0.73 \pm 0.05 M_{\text {Jup }}$. In both of these systems, including the additional planets in the fitting process significantly reduced the eccentricity of the original planet. These systems are thus examples of how multiple-planet systems can masquerade as moderately eccentric single-planet systems.
\end{abstract}

Key words: planetary systems - stars: individual (HD 142, HD 159868) - techniques: radial velocities

Online-only material: color figures

\section{INTRODUCTION}

Recent discoveries of multi-planet systems from maturing high-precision Doppler planet searches are revealing a surprising diversity of planetary system properties. It is becoming apparent that, given a sufficient number of high-precision observations, many seemingly solitary stars or single-planet systems are found to host additional orbiting bodies. This trend is evident both at extremely low masses (e.g., Anglada-Escudé et al. 2012; Mayor et al. 2011; Vogt et al. 2010) and long periods (e.g., HD 134987c, Jones et al. 2010; 47 UMa d, Gregory \& Fischer 2010).

Confirmation by independent observatories is extremely useful when testing the potential detection of additional planets, particularly if they have small radial-velocity amplitudes or the host star has a high level of velocity jitter. For example, Bean et al. (2008) reported a third planet in the HD 74156 system using data from the Hobby-Eberly Telescope. However, using the same spectra processed with two different, independent Doppler velocity codes, Wittenmyer et al. (2009) could not confirm that planet. The Keck observations presented by Meschiari et al. (2011) were also inconsistent with a third planet in that system. High-precision and high-cadence data from multiple sites have proved critical in the confirmation of low-mass planets such as HD 4308 (Udry et al. 2006; O'Toole et al. 2009), the 61 Vir three-planet system (Vogt et al. 2010), and HD 114613. For candidate multiple-planet systems, dynamical stability modeling is also a critical tool, since periodic signals arising from observational sampling or stellar activity can be misinterpreted as planets. The inclusion of rigorous dynamical modeling has recently shown some candidate planetary systems to be unfeasible, e.g., Horner et al. (2011), Tuomi (2011), and Wittenmyer et al. (2012).

In this work, we present new data from the Anglo-Australian Planet Search (AAPS) which provide evidence for one additional planet orbiting HD 142 (Section 3.1) with a period of 6005 days. We also perform a Bayesian analysis (Section 3.2), yielding results that are in agreement with the conventional least-squares solution. In Section 3.3, we present AAPS and Keck data indicating a second planet in the HD 159868 system with an orbital period of 352 days. These two proposed multiplanet systems are subjected to detailed dynamical testing in Section 4. In Section 5, we use direct imaging of the HD 142 system to rule out the known stellar companion as the source of the observed radial-velocity signal. Finally, in Section 6, we place these discoveries in the context of the overall distribution of exoplanet properties and give our conclusions.

\section{OBSERVATIONS AND STELLAR PARAMETERS}

AAPS Doppler measurements are made with the UCLES echelle spectrograph (Diego et al. 1991). Keck Doppler measurements are made with the High Resolution Echelle Spectrometer spectrograph (Vogt et al. 1994). An iodine absorption cell provides wavelength calibration from 5000 to $6200 \AA$. The spectrograph point-spread function (PSF) and wavelength calibration are derived from the iodine absorption lines embedded on every pixel of the spectrum by the cell (Valenti et al. 1995; Butler et al. 1996). The result is a precision Doppler velocity estimate for each epoch, along with an internal uncertainty estimate, which includes the effects of photon-counting uncertainties, residual errors in the spectrograph PSF model, and variation in the underlying spectrum between the iodine-free template and epoch spectra observed through the iodine cell. All velocities are measured relative to the zero point defined by the template observation.

HD 142 has been observed by the Anglo-Australian Telescope (AAT) at 82 epochs, with a total data span of 5067 days. HD 159868 has been observed by the AAT at 47 epochs, with a total data span of 3396 days. In this analysis, we also add 
Table 1

AAT Radial Velocities for HD 142

\begin{tabular}{|c|c|c|}
\hline JD-2,400,000 & $\begin{array}{l}\text { Velocity } \\
\left(\mathrm{m} \mathrm{s}^{-1}\right)\end{array}$ & $\begin{array}{l}\text { Uncertainty } \\
\quad\left(\mathrm{m} \mathrm{s}^{-1}\right)\end{array}$ \\
\hline 50830.95872 & 29.8 & 3.2 \\
\hline 51121.01944 & 36.9 & 3.6 \\
\hline 51385.31047 & 84.7 & 5.9 \\
\hline 51411.20252 & 75.5 & 6.3 \\
\hline 51473.08503 & 33.9 & 3.3 \\
\hline 51525.92509 & 54.0 & 4.0 \\
\hline 51526.95290 & 46.3 & 2.9 \\
\hline 51683.33138 & 86.4 & 3.8 \\
\hline 51743.27654 & 85.3 & 3.4 \\
\hline 51745.26417 & 90.7 & 5.5 \\
\hline 51767.26990 & 63.9 & 3.6 \\
\hline 51768.25417 & 66.1 & 3.2 \\
\hline 51828.06072 & 40.4 & 3.9 \\
\hline 51856.06429 & 12.0 & 5.5 \\
\hline 51856.92498 & 46.3 & 7.4 \\
\hline 51918.94072 & 55.5 & 3.8 \\
\hline 52061.29661 & 84.6 & 3.4 \\
\hline 52092.26831 & 70.0 & 3.3 \\
\hline 52093.28756 & 59.4 & 3.3 \\
\hline 52127.22295 & 34.6 & 4.1 \\
\hline 52128.15455 & 42.4 & 3.9 \\
\hline 52130.24335 & 35.4 & 3.6 \\
\hline 52151.21126 & 31.6 & 2.9 \\
\hline 52152.07857 & 36.5 & 3.7 \\
\hline 52154.15414 & 28.8 & 3.3 \\
\hline 52187.09998 & 34.9 & 2.9 \\
\hline 52188.03596 & 30.0 & 2.9 \\
\hline 52189.01990 & 22.3 & 3.0 \\
\hline 52190.00244 & 29.8 & 2.9 \\
\hline 52423.32977 & 58.3 & 3.1 \\
\hline 52425.33759 & 38.8 & 3.2 \\
\hline 52456.32088 & 15.8 & 3.5 \\
\hline 52477.24794 & 25.6 & 3.4 \\
\hline 52511.09845 & 37.8 & 3.4 \\
\hline 52654.91588 & 59.7 & 4.7 \\
\hline 52784.32722 & 36.4 & 3.4 \\
\hline 52857.23832 & 24.1 & 2.3 \\
\hline 52861.30634 & 24.1 & 5.1 \\
\hline 52946.03964 & 27.6 & 3.8 \\
\hline 53007.97796 & 35.1 & 3.9 \\
\hline 53041.95410 & 72.0 & 4.4 \\
\hline 53042.90882 & 64.9 & 4.9 \\
\hline 53215.27934 & -7.3 & 2.6 \\
\hline 53243.28286 & 6.6 & 3.7 \\
\hline 53244.22676 & 0.4 & 3.8 \\
\hline 53246.09497 & 4.5 & 2.9 \\
\hline 53281.13767 & -0.8 & 3.6 \\
\hline 53509.33429 & 4.5 & 2.0 \\
\hline 53516.33116 & 2.5 & 2.3 \\
\hline 53570.30140 & -17.4 & 1.8 \\
\hline 53576.22037 & -8.8 & 1.7 \\
\hline 53579.27530 & -11.6 & 1.7 \\
\hline 53632.19723 & -41.2 & 1.9 \\
\hline 53942.21660 & -33.9 & 1.6 \\
\hline 54008.12359 & -22.8 & 2.2 \\
\hline 54013.13830 & -11.5 & 1.7 \\
\hline 54016.18494 & -22.3 & 1.8 \\
\hline 54038.10169 & -12.5 & 1.8 \\
\hline 54120.92520 & 22.7 & 2.1 \\
\hline 54255.26045 & -32.8 & 2.5 \\
\hline 54334.13051 & -43.4 & 2.5 \\
\hline 54374.14271 & -24.4 & 2.1 \\
\hline 54428.94253 & -3.0 & 1.7 \\
\hline 54780.10864 & -3.4 & 1.5 \\
\hline 55076.28334 & -58.7 & 2.1 \\
\hline
\end{tabular}

Table 1

(Continued)

\begin{tabular}{lrc}
\hline \hline JD-2,400,000 & $\begin{array}{r}\text { Velocity } \\
\left(\mathrm{m} \mathrm{s}^{-1}\right)\end{array}$ & $\begin{array}{c}\text { Uncertainty } \\
\left(\mathrm{m} \mathrm{s}^{-1}\right)\end{array}$ \\
\hline 55101.11333 & -19.8 & 4.2 \\
55170.91268 & -7.3 & 2.0 \\
55376.32957 & -89.9 & 3.8 \\
55377.32745 & -86.0 & 2.7 \\
55401.20729 & -59.7 & 2.3 \\
55428.24962 & -16.4 & 4.0 \\
55457.13465 & -16.3 & 2.5 \\
55518.99948 & 32.5 & 2.3 \\
55521.00990 & 10.7 & 2.6 \\
55751.31147 & -31.3 & 3.6 \\
55757.24366 & -34.3 & 2.7 \\
55786.25163 & 0.7 & 3.2 \\
55788.32904 & -22.7 & 3.2 \\
55845.10610 & 14.7 & 2.6 \\
55874.03173 & 20.5 & 3.0 \\
55874.98064 & 15.4 & 3.6 \\
55897.91075 & 7.6 & 2.3 \\
\hline
\end{tabular}

34 Keck epochs spanning 1593 days. The radial-velocity data for HD 142 are presented in Table 1, and the data for HD 159868 are in Tables 2 and 3. The physical parameters of HD 142 are given in Tinney et al. (2002), and those for HD 159868 are given in O'Toole et al. (2007). Tables 4 and 5 summarize the parameters for HD 142 and HD 159868, respectively. Briefly, HD 142 is a G1 IV star with a mass of $1.15 \pm 0.10 M_{\odot}$, is possibly slightly evolved (Tinney et al. 2002), is chromospherically inactive $\left(\log R_{H K}^{\prime}=-4.95\right)$, and has a moderately rapid rotation rate $\left(V \sin i=10.4 \mathrm{~km} \mathrm{~s}^{-1}\right)$. HD 159868 is a G5 dwarf with a mass of $1.087_{-0.033}^{+0.032} M_{\odot}$, solar metallicity, and is a chromospherically inactive $\left(\log R_{H K}^{\prime}=-4.96\right)$ slow rotator $\left(V \sin i=2.1 \mathrm{~km} \mathrm{~s}^{-1}\right)$. HD 142 has a known stellar companion (Poveda et al. 1994), which is a late $\mathrm{K} /$ early $\mathrm{M}$ star with a mass of $\sim 0.56 M_{\odot}$ (Eggenberger et al. 2007; Raghavan et al. 2006) and a projected separation of 105.1 AU.

\section{ORBIT FITTING AND PLANETARY PARAMETERS}

\section{1. $H D 142$}

The Jupiter-mass planet orbiting HD 142 with a period of 339 days was one of the first discoveries reported by the AAPS (Tinney et al. 2002). A further 10 years of observations have revealed evidence for a long-period signal consistent with a second planet in the system. In the discovery paper, the oneplanet fit had a root-mean-square (rms) residual scatter of $5.9 \mathrm{~m} \mathrm{~s}^{-1}$, consistent with the $3-4 \mathrm{~m} \mathrm{~s}^{-1}$ estimated jitter for that star. Using the formulation of Wright (2005), we now estimate a jitter of $4.5 \mathrm{~m} \mathrm{~s}^{-1}$, which we apply in quadrature to the internal uncertainties shown in Table 1. A one-planet fit to the current data set for HD 142 now has an rms of $31.1 \mathrm{~m} \mathrm{~s}^{-1}$. This significantly exceeds both the scatter due to the underlying precision of our AAPS Doppler measurement system and the predicted levels of stellar activity jitter expected for HD 142. This excess scatter led us to investigate the possibility of one or more additional planets orbiting this star.

Visual inspection of the residuals to the one-planet fit shows an obvious long-period signal $(P>1000$ days), and the periodogram shows a significant peak at very long periods (Figure 1), so we proceed to fit a second planet. First, we explored the vast and uncertain parameter space of the long- 
Table 2

AAT Radial Velocities for HD 159868

\begin{tabular}{|c|c|c|}
\hline JD-2,400,000 & $\begin{array}{l}\text { Velocity } \\
\left(\mathrm{m} \mathrm{s}^{-1}\right)\end{array}$ & $\begin{array}{c}\text { Uncertainty } \\
\left(\mathrm{m} \mathrm{s}^{-1}\right)\end{array}$ \\
\hline 52390.22780 & 29.1 & 1.4 \\
\hline 52422.14712 & 10.2 & 1.4 \\
\hline 52453.04281 & 11.9 & 1.5 \\
\hline 52456.07120 & 20.2 & 1.6 \\
\hline 52477.02060 & 8.1 & 1.5 \\
\hline 52711.26892 & -29.3 & 2.9 \\
\hline 52747.25274 & -34.7 & 1.5 \\
\hline 52751.26030 & -34.4 & 1.4 \\
\hline 52786.09989 & -59.4 & 1.3 \\
\hline 52858.95394 & -25.3 & 1.3 \\
\hline 52942.93463 & -17.4 & 1.9 \\
\hline 53214.09869 & 8.2 & 1.8 \\
\hline 53216.04487 & 9.1 & 1.4 \\
\hline 53242.96824 & 20.6 & 1.3 \\
\hline 53484.23874 & 31.4 & 1.3 \\
\hline 53486.16508 & 35.1 & 1.4 \\
\hline 53510.17399 & 18.3 & 1.4 \\
\hline 53521.19560 & 17.8 & 1.4 \\
\hline 53572.06973 & 34.9 & 1.3 \\
\hline 53631.89779 & 49.5 & 1.2 \\
\hline 53842.24103 & -31.5 & 1.5 \\
\hline 53939.00914 & -16.8 & 1.3 \\
\hline 53947.05674 & -21.3 & 1.2 \\
\hline 54008.91766 & -9.9 & 0.9 \\
\hline 54011.90938 & -5.1 & 1.4 \\
\hline 54015.94916 & -9.4 & 1.1 \\
\hline 54017.89861 & -3.0 & 1.3 \\
\hline 54037.89603 & -9.0 & 1.4 \\
\hline 54224.26216 & -40.6 & 1.2 \\
\hline 54227.16135 & -28.6 & 1.3 \\
\hline 54255.03411 & -27.9 & 1.2 \\
\hline 54371.88740 & 25.7 & 1.1 \\
\hline 54553.20262 & 18.1 & 1.6 \\
\hline 54907.25523 & 4.8 & 1.7 \\
\hline 55101.91055 & 1.3 & 1.2 \\
\hline 55104.94555 & -10.7 & 1.4 \\
\hline 55109.96044 & -22.7 & 1.3 \\
\hline 55313.25667 & -52.9 & 1.4 \\
\hline 55317.16029 & -35.3 & 1.5 \\
\hline 55376.12410 & -16.8 & 1.5 \\
\hline 55399.06643 & -7.1 & 1.4 \\
\hline 55429.85887 & 6.8 & 1.4 \\
\hline 55456.87174 & 4.8 & 1.7 \\
\hline 55664.29971 & 19.6 & 1.3 \\
\hline 55692.24347 & 36.7 & 1.5 \\
\hline 55756.91450 & 74.4 & 1.9 \\
\hline 55786.07264 & 65.2 & 1.9 \\
\hline
\end{tabular}

period signal with a genetic algorithm (e.g., Cochran et al. 2007; Tinney et al. 2011; Wittenmyer et al. 2012). We allowed the second planet to take on periods between 1000 and 10,000 days, and an eccentricity $e<0.6$. The genetic algorithm ran for 50,000 iterations, each of which consisted of typically 1000-3000 generations, during which the two-planet fits evolved toward a $\chi^{2}$ minimum. The best-fit system parameters from this process are thus the result of $\sim 10^{8}$ trial Keplerian fits. Used in this way, the genetic algorithm is an effective way of exploring a large parameter space, which is particularly important when the candidate planet's period is comparable to the length of the available data. We then used the GaussFit least-squares fitting code (Jefferys et al. 1987) to obtain a Keplerian model fit, with the best two-planet fit parameters from the genetic selection as initial inputs.
Table 3

Keck Radial Velocities for HD 159868

\begin{tabular}{|c|c|c|}
\hline JD-2,400,000 & $\begin{array}{l}\text { Velocity } \\
\left(\mathrm{m} \mathrm{s}^{-1}\right)\end{array}$ & $\begin{array}{c}\text { Uncertainty } \\
\left(\mathrm{m} \mathrm{s}^{-1}\right)\end{array}$ \\
\hline 54246.99415 & -39.3 & 0.8 \\
\hline 54247.97421 & -32.3 & 1.1 \\
\hline 54248.92666 & -35.4 & 1.1 \\
\hline 54251.95192 & -24.7 & 1.1 \\
\hline 54255.97421 & -24.2 & 0.8 \\
\hline 54277.88461 & -17.7 & 1.2 \\
\hline 54278.93840 & -13.8 & 1.1 \\
\hline 54304.82235 & 2.3 & 0.8 \\
\hline 54305.83320 & 6.3 & 0.7 \\
\hline 54306.82686 & 0.0 & 1.0 \\
\hline 54307.86512 & 3.6 & 0.7 \\
\hline 54308.85011 & -0.6 & 0.7 \\
\hline 54309.83390 & -1.1 & 0.7 \\
\hline 54310.82821 & 6.4 & 0.7 \\
\hline 54311.82166 & 9.2 & 0.7 \\
\hline 54312.81832 & 8.5 & 0.7 \\
\hline 54313.79003 & 9.7 & 0.7 \\
\hline 54314.79189 & 8.9 & 0.7 \\
\hline 54335.72425 & 20.8 & 1.1 \\
\hline 54601.91517 & 24.8 & 1.1 \\
\hline 55024.86442 & -2.8 & 0.6 \\
\hline 55049.83900 & -11.9 & 0.8 \\
\hline 55052.81141 & -0.7 & 0.6 \\
\hline 55260.15738 & -60.9 & 1.0 \\
\hline 55369.98576 & -8.6 & 0.6 \\
\hline 55409.84249 & -10.9 & 0.6 \\
\hline 55462.73665 & -2.1 & 0.7 \\
\hline 55638.13563 & -1.6 & 0.8 \\
\hline 55665.10001 & 14.3 & 0.7 \\
\hline 55670.06654 & 14.8 & 0.7 \\
\hline 55720.04108 & 48.9 & 0.7 \\
\hline 55750.87325 & 56.1 & 1.3 \\
\hline 55825.74817 & 40.1 & 0.7 \\
\hline 55839.72241 & 35.0 & 1.2 \\
\hline
\end{tabular}

The two-planet fit has an rms of $11.2 \mathrm{~m} \mathrm{~s}^{-1}$, which is still somewhat higher than that expected for this star. A periodogram of the residuals shows a peak at 108 days (Figure 1). We used a bootstrap randomization process (Kürster et al. 1997) to assess the false-alarm probability of the peak at 108 days. The bootstrap method randomly shuffles the velocity observations while keeping the times of observation fixed. The periodogram of this shuffled data set is then computed and its highest peak recorded. From 10,000 such realizations, the peak at 108 days has a bootstrap false-alarm probability of $5.1 \%$. In mid-2011, this false-alarm probability was $2.5 \%$ - that the addition of new data did not improve the statistical credibility of the 108 day signal leads us to conclude that the signal cannot be claimed as planetary in origin at this time.

The residual scatter about the two-planet fit remains much higher than expected given the jitter estimate of $4.45 \mathrm{~m} \mathrm{~s}^{-1}$ for HD 142. However, the estimation of stellar activity jitter is a rather imprecise process, with uncertainties up to a factor of two (J. Wright 2008, private communication). Hence, it is possible that we have underestimated the activity jitter for HD 142. A jitter estimate of $11.3 \mathrm{~m} \mathrm{~s}^{-1}$ is required to produce a reduced $\chi^{2}$ of unity for the two-planet fit. Examining the distribution of jitter estimates for similar stars (Wright 2005; top panel of their Figure 7), the distribution has a tail extending toward a maximum jitter of $11 \mathrm{~m} \mathrm{~s}^{-1}$, but only includes 36 stars in total. Isaacson \& Fischer (2010) provide a different formulation to 

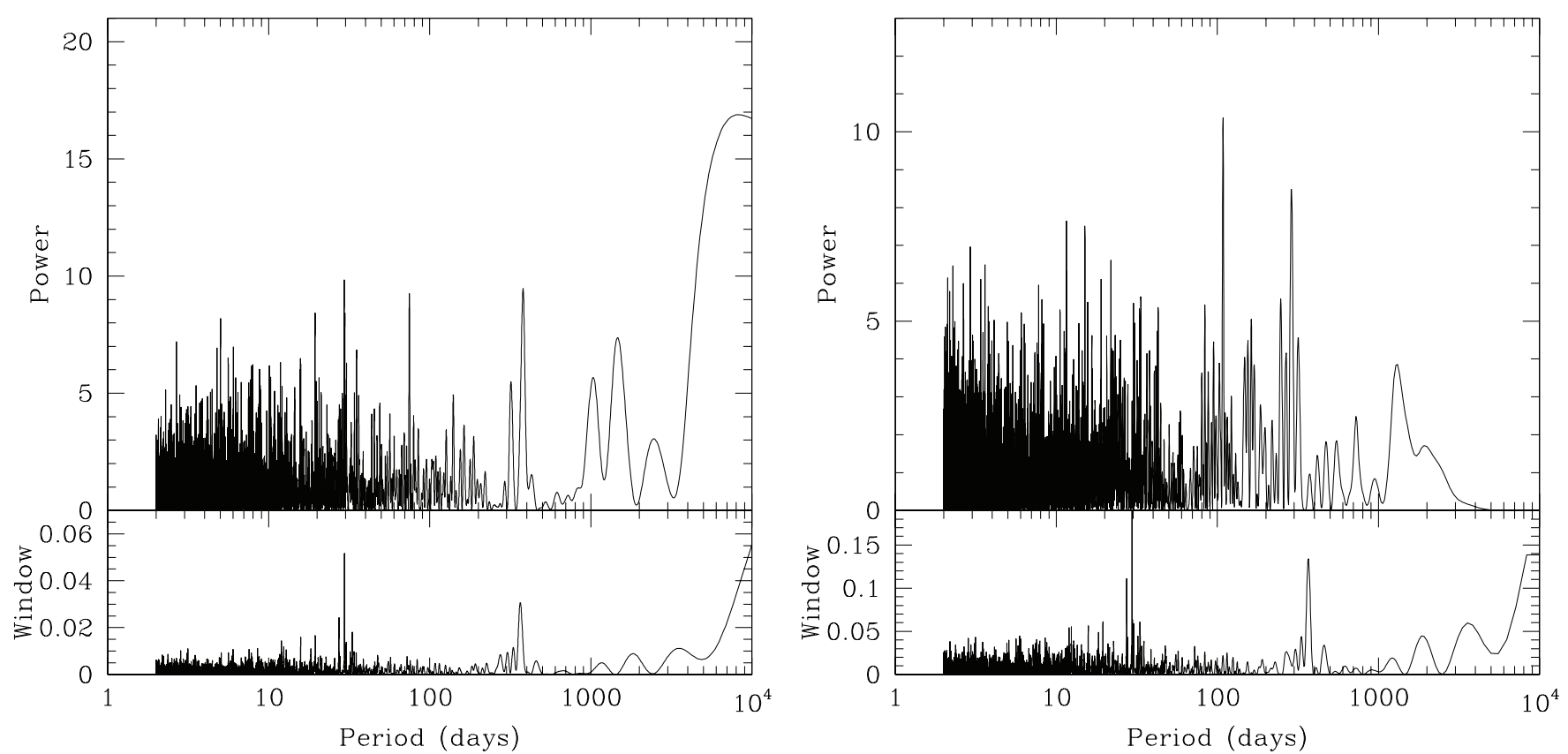

Figure 1. Left panel: periodogram of residuals for HD 142 after fitting one planet at $P=350$ days. A long-period signal is clearly present. Right panel: periodogram of residuals for HD 142 after fitting two planets, at $P=350$ and 6005 days. The highest remaining peak is at a period of 108 days, with a false-alarm probability of $5.1 \%$.

Table 4

Stellar Parameters for HD 142

\begin{tabular}{|c|c|c|}
\hline Parameter & Value & Reference \\
\hline \multirow[t]{2}{*}{ Spec. type } & G1 IV & Tinney et al. (2002) \\
\hline & F7 V & Gray (2006) \\
\hline \multirow[t]{2}{*}{ Mass $\left(M_{\odot}\right)$} & $1.15 \pm 0.10$ & Tinney et al. (2002) \\
\hline & $1.232_{-0.16}^{+0.22}$ & Takeda et al. (2007) \\
\hline Distance (pc) & $25.7 \pm 0.3$ & van Leeuwen (2007) \\
\hline$M_{V}$ & 3.66 & \\
\hline \multirow[t]{3}{*}{ Radius $\left(R_{\odot}\right)$} & $1.47 \pm 0.04$ & van Belle \& von Braun (2009) \\
\hline & $1.40 \pm 0.05$ & Takeda et al. (2007) \\
\hline & $1.43 \pm 0.07$ & Lang (1980) \\
\hline \multirow[t]{2}{*}{$V \sin i\left(\mathrm{~km} \mathrm{~s}^{-1}\right)$} & $10.4 \pm 0.5$ & Valenti \& Fischer (2005) \\
\hline & $10.35 \pm 0.50$ & Butler et al. (2006) \\
\hline \multirow[t]{2}{*}{$\log R_{H K}^{\prime}$} & -4.92 & Tinney et al. (2002) \\
\hline & -4.95 & Jenkins et al. (2006) \\
\hline \multirow[t]{5}{*}[\mathrm{Fe}/\mathrm{H}]{} & $0.10 \pm 0.03$ & Valenti \& Fischer (2005) \\
\hline & $0.09 \pm 0.05$ & Sousa et al. (2008) \\
\hline & $-0.02 \pm 0.07$ & Bond et al. (2006) \\
\hline & $0.117 \pm 0.070$ & Gonzalez \& Laws (2007) \\
\hline & $-0.02 \pm 0.06$ & Ramírez et al. (2007) \\
\hline \multirow[t]{5}{*}{$T_{\text {eff }}(\mathrm{K})$} & $6403 \pm 65$ & Sousa et al. (2008) \\
\hline & $6150 \pm 35$ & Bond et al. (2006) \\
\hline & $6245 \pm 48$ & Malyuto \& Shvelidze (2011) \\
\hline & 6249 & Valenti \& Fischer (2005) \\
\hline & 6170 & Randich et al. (1999) \\
\hline \multirow[t]{4}{*}{$\log g$} & $4.62 \pm 0.07$ & Sousa et al. (2008) \\
\hline & $4.26_{-0.2}^{+0.3}$ & Takeda et al. (2007) \\
\hline & 4.19 & Valenti \& Fischer (2005) \\
\hline & 4.2 & Randich et al. (1999) \\
\hline
\end{tabular}

estimate activity jitter, using the CaII S-index. The Mount Wilson S-index for HD 142 is $S_{\mathrm{MW}}=0.187$ (Jenkins et al. 2006). Using the Isaacson \& Fischer (2010) formulation, this yields an estimated jitter of $3.07 \mathrm{~m} \mathrm{~s}^{-1}$. Based on the chromospheric activity index and the high $\log g$ (Table 4), it appears that HD 142 is an inactive dwarf star, which nonetheless presents a high level of radial-velocity noise. We note that this star is quite
Table 5

Stellar Parameters for HD 159868

\begin{tabular}{lcl}
\hline \hline Parameter & Value & Reference \\
\hline Spec. type & G5 V & Houk (1978) \\
Mass $\left(M_{\odot}\right)$ & $1.087_{-0.033}^{+0.032}$ & Takeda et al. (2007) \\
& $1.16_{-0.18}^{+0.27}$ & Valenti \& Fischer (2005) \\
& 0.919 & Sousa et al. (2008) \\
Distance $(\mathrm{pc})$ & $52.7 \pm 3.0$ & Perryman et al. (1997) \\
$M_{V}$ & 3.63 & \\
$V \sin i\left(\mathrm{~km} \mathrm{~s}^{-1}\right)$ & 2.1 & Valenti \& Fischer (2005) \\
$\log R_{H K}^{\prime}$ & -4.96 & Jenkins et al. (2006) \\
{$[\mathrm{Fe} / \mathrm{H}]$} & 0.00 & Valenti \& Fischer (2005) \\
& $-0.08 \pm 0.01$ & Sousa et al. (2008) \\
$T_{\mathrm{eff}}(\mathrm{K})$ & 5623 & Valenti \& Fischer (2005) \\
& $5558 \pm 15$ & Sousa et al. (2008) \\
$\log g$ & $3.99_{-0.04}^{+0.05}$ & Takeda et al. (2007) \\
& 3.92 & Valenti \& Fischer (2005) \\
& $3.96 \pm 0.02$ & Sousa et al. (2008) \\
\hline
\end{tabular}

a rapid rotator: it has a $V \sin i$ of $10.4 \mathrm{~km} \mathrm{~s}^{-1}$, compared to typical planet-search targets which have $V \sin i$ of $2-4 \mathrm{~km} \mathrm{~s}^{-1}$. We conclude that the poor velocity precision for HD 142 is attributable to the rapid rotation, which broadens the spectral lines and limits our ability to derive extremely precise radial velocities.

Using a stellar mass of $1.15 \pm 0.10 M_{\odot}$ (Tinney et al. 2002), we estimate the minimum mass, $m \sin i$, for the outermost planet (planet c) to be $5.3 \pm 0.7 M_{\text {Jup }}$. The two-planet fit is shown in Figure 2 and the planetary parameters are given in Table 6 . The individual fits for each of the two planets are shown in Figure 3.

\subsection{A Bayesian Analysis for HD 142}

Thirteen years of AAT data have provided evidence for a very long-period planet (HD 142c) with a period of more than 6000 days. The baseline of these AAT observations is the longest currently available at high precision for this star, which makes 


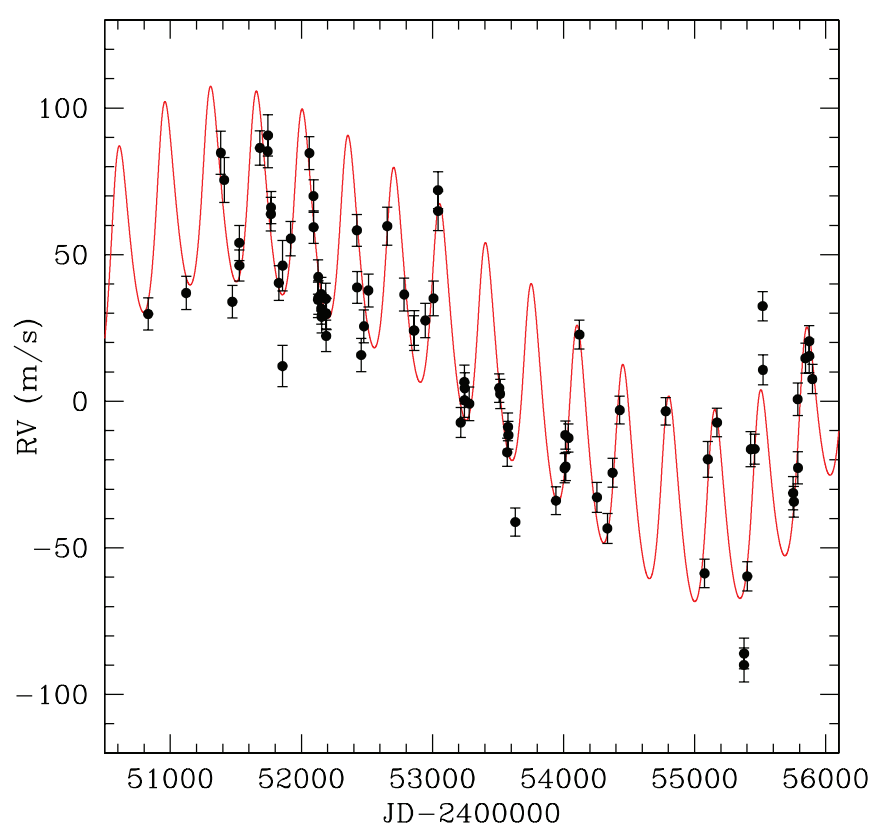

Figure 2. Two-planet fit for HD 142. The residuals of this fit are $11.2 \mathrm{~m} \mathrm{~s}^{-1}$, and no further significant signals are present.

(A color version of this figure is available in the online journal.)

independent confirmation of HD $142 \mathrm{c}$ problematic. A third candidate signal is present, with a period of 108 days and a velocity semiamplitude $K \sim 12 \mathrm{~m} \mathrm{~s}^{-1}$, which is comparable to the $11.2 \mathrm{~m} \mathrm{~s}^{-1}$ residual velocity scatter about the two-planet fit. It is prudent, then, to employ an independent analysis to test the plausibility of the 108 day signal.

We analyzed the AAPS radial velocities using posterior samplings of different models and the comparisons of these models using Bayesian model probabilities (e.g., Tuomi \& Kotiranta 2009; Tuomi 2011; Tuomi et al. 2011). We used the adaptive Metropolis algorithm (Haario et al. 2001) for posterior samplings because it appears to be a reasonably efficient method for analyzing radial velocities with Keplerian models (Tuomi 2011; Tuomi et al. 2011). We present the results using the maximum a posteriori (MAP) estimates and the corresponding $99 \%$ credibility intervals $\left(\mathcal{D}_{0.99}\right)$, i.e., the Bayesian credibility sets as defined in Tuomi \& Kotiranta (2009). Our prior probability densities of the model parameters are those used in Ford \& Gregory (2007), with slight modifications. We penalized very high eccentricities by setting the prior densities for orbital eccentricities $\pi(e) \propto \mathcal{N}\left(0, \sigma_{e}^{2}\right)$, where the parameter $\sigma_{e}$ was set to have a value of 0.3 that still allows the orbital eccentricities to have high values if the data insist so. We also adopted conservative prior probabilities for models with $k$ Keplerian signals such that $P\left(\mathcal{M}_{k}\right)=2 P\left(\mathcal{M}_{k+1}\right)$, i.e., that the prior probability of having $k+1$ planets in the system is always two times less than having $k$ planets. Essentially, this enables us to be more confident with the interpretation of our model probabilities-if there appear to be $k$ Keplerian signals in the data, we actually underestimate the significance of the weakest signal because of these priors.

The probabilities of models $\mathcal{M}_{k}, k=0, \ldots, 3$ planets and the corresponding rms values are shown in Table 7 . The velocity jitter was also included as a free parameter in the Bayesian model, and the best-fit jitter values are given in Table 7 for each of the $k$-planet models. These results support the presence of three Keplerian signals in the data, although the parameters of the 108 day signal ("planet d") are poorly constrained. The orbital parameters of this three-planet solution are shown in Table 8. The distribution of allowed orbital periods for the outer planet had a substantially longer tail toward longer periods (as expected given the data span is shorter than the expected orbital period for this planet). The large uncertainty in the period of planet $\mathrm{c}$ maps directly into large uncertainties for the eccentricity and velocity semiamplitude $K$. We therefore note that the nominal $1 \sigma$ uncertainties in the parameters of planet $\mathrm{c}$ as given in Table 6 are likely underestimated, and we advise the reader to consider the $99 \%$ confidence intervals in Table 8 as more comprehensive.

\section{3. $H D 159868$}

O'Toole et al. (2007) reported the detection of a longperiod ( $P=986$ days), eccentric $(e=0.69)$ planet orbiting HD 159868, based on 4.5 years of AAT data. That fit had a residual rms scatter of $8.4 \mathrm{~m} \mathrm{~s}^{-1}$, which the authors noted as larger than expected for that star. They speculated that a second planet with $P=180$ days would substantially reduce the rms but, wary of the sampling difficulties in constraining planet candidates with periods near one-half of a sidereal year, they presented only the single-planet solution for HD 159868. Now, with nearly twice as much data (Table 2), the single-planet fit has an rms of $15.8 \mathrm{~m} \mathrm{~s}^{-1}$. As is the case for HD 142 above, the worsening single-planet fit gives a clue that additional planets are present in this system. Here we adopt a jitter estimate of $2.65 \mathrm{~m} \mathrm{~s}^{-1}$, and apply this in quadrature to the uncertainties given in Table 2 before performing orbital fitting. The fitting procedures used followed those outlined above for HD 142.

Interestingly, a one-planet fit for HD 159868 now has an eccentricity of only $0.16 \pm 0.11$, which is markedly different from the $e=0.69$ solution presented in O'Toole et al. (2007). A periodogram of the residuals to this fit (Figure 4) shows a large peak at 355 days. This peak has a bootstrap false-alarm probability $<0.01 \%$. Now armed with substantial evidence for a second planet in the system, we proceed with a two-Keplerian solution. For the final orbit fit, we also include 4.3 years of data from the Keck telescope. The best fit has a second planet with a period of $352.3 \pm 1.3$ days (Table 6); adopting a stellar mass of $1.087_{-0.033}^{+0.032}$ (Takeda et al. 2007), the planet has $m \sin i=0.73 \pm 0.05 M_{\mathrm{Jup}}$. Figure 5 shows the two-planet fit and the phase coverage for the new planet candidate, which has a period near one year. This fit has a total residual rms of $5.8 \mathrm{~m} \mathrm{~s}^{-1}$ (AAT: $6.7 \mathrm{~m} \mathrm{~s}^{-1}$; Keck: $4.6 \mathrm{~m} \mathrm{~s}^{-1}$ ), which is still somewhat higher than expected based on the instrumental noise and stellar activity jitter. Based on the somewhat low $\log g$ values given in Table 5, HD 159868 may be slightly evolved. If HD 159868 is a subgiant, the velocity jitter may be closer to $\sim 5 \mathrm{~m} \mathrm{~s}^{-1}$, typical of subgiants (Kjeldsen \& Bedding 1995; Johnson et al. 2010; Wittenmyer et al. 2011a) and consistent with the rms scatter about our two-planet fit.

A periodogram of the residuals now has a peak at 12.6 days, but it is not significant, with a bootstrap false-alarm probability of $27 \%$. As a further check, we also performed a Bayesian analysis as described above for the HD 142 system, and we obtain model probabilities (Table 9) which confidently indicate two Keplerian signals. These results demonstrate how two planets in nearly circular orbits can mimic a single eccentric planet when data are sparse and more subject to vagaries of sampling. This can cause signals near one year to be missed (Tinney et al. 2011; Anglada-Escudé et al. 2010). 

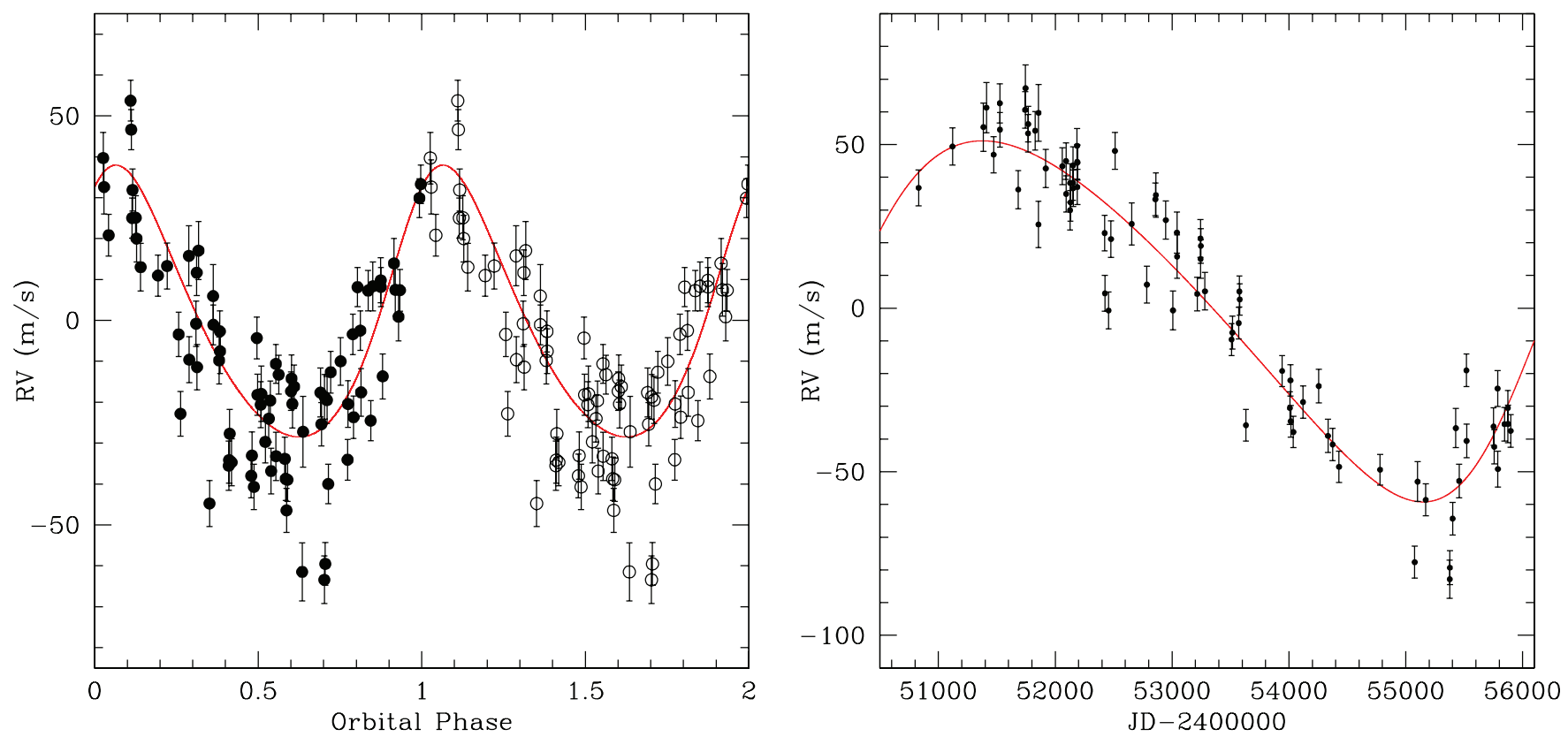

Figure 3. Left panel: fit for HD $142 \mathrm{~b}$, the previously known planet with $P=350$ days. Two cycles are shown for clarity. Right panel: fit for HD $142 \mathrm{c}$, with $P=6005$ days. In both panels, the signal of the other planet has been removed.

(A color version of this figure is available in the online journal.)

Table 6

Keplerian Orbital Solutions

\begin{tabular}{lccccccc}
\hline \hline Planet & $\begin{array}{c}\text { Period } \\
(\text { days })\end{array}$ & $\begin{array}{c}T_{0} \\
(\mathrm{JD}-2,400,000)\end{array}$ & $e$ & $\begin{array}{c}\omega \\
(\mathrm{deg})\end{array}$ & $\begin{array}{c}K \\
\left(\mathrm{~m} \mathrm{~s}^{-1}\right)\end{array}$ & $\begin{array}{c}m \sin i \\
\left(M_{\text {Jup }}\right)\end{array}$ & $\begin{array}{c}a \\
(\mathrm{AU})\end{array}$ \\
\hline HD 142b & $349.7 \pm 1.2$ & $52683 \pm 26$ & $0.17 \pm 0.06$ & $327 \pm 26$ & $33.2 \pm 2.5$ & $1.25 \pm 0.15$ & $1.02 \pm 0.03$ \\
HD 142c & $6005 \pm 477$ & $55954 \pm 223$ & $0.21 \pm 0.07$ & $250 \pm 20$ & $55.2 \pm 3.0$ & $5.3 \pm 0.7$ & $6.8 \pm 0.5$ \\
HD 159868b & $1178.4 \pm 8.8$ & $53435 \pm 56$ & $0.01 \pm 0.03$ & $350 \pm 171$ & $38.3 \pm 1.1$ & $2.10 \pm 0.11$ & $2.25 \pm 0.03$ \\
HD 159868c & $352.3 \pm 1.3$ & $53239 \pm 21$ & $0.15 \pm 0.05$ & $290 \pm 25$ & $20.1 \pm 1.1$ & $0.73 \pm 0.05$ & $1.00 \pm 0.01$ \\
\hline
\end{tabular}

Table 7

The Relative Posterior Probabilities of Models $\mathcal{M}_{k}$ with $k=0, \ldots, 3$ Keplerian Signals Given the AAT Data for HD 142

\begin{tabular}{cccc}
\hline \hline$k$ & $P\left(\mathcal{M}_{k} \mid d\right)$ & $\begin{array}{c}\text { Jitter } \\
\left(\mathrm{m} \mathrm{s}^{-1}\right)\end{array}$ & $\begin{array}{c}\mathrm{rms} \\
\left(\mathrm{m} \mathrm{s}^{-1}\right)\end{array}$ \\
\hline 0 & $1.5 \times 10^{-48}$ & 39.7 & 39.1 \\
1 & $1.2 \times 10^{-32}$ & 23.7 & 22.5 \\
2 & $1.4 \times 10^{-8}$ & 11.2 & 11.2 \\
3 & $\sim 1$ & 8.8 & 8.6 \\
\hline
\end{tabular}

Notes. The velocity jitter was also fitted as a free parameter in the model.

\section{DYNAMICAL STABILITY ANALYSIS}

While single-planet systems can be fully solved with a simple Keplerian analysis, gravitationally interacting systems of multiple planets require a full Newtonian analysis. Proposed solutions need to be shown to be dynamically stable over reasonably long timescales (Horner et al. 2011, 2012; Wittenmyer et al. 2012) to be considered "real." Gravitationally interacting systems provide both an independent check on "reality" (Fabrycky et al. 2012) and a useful means to constrain or solve for the orbital inclination angle and true mass of the planets (Rivera et al. 2005).

\subsection{The HD 142 System}

To examine the dynamical stability of the proposed HD 142 planetary system, we performed a series of highly detailed

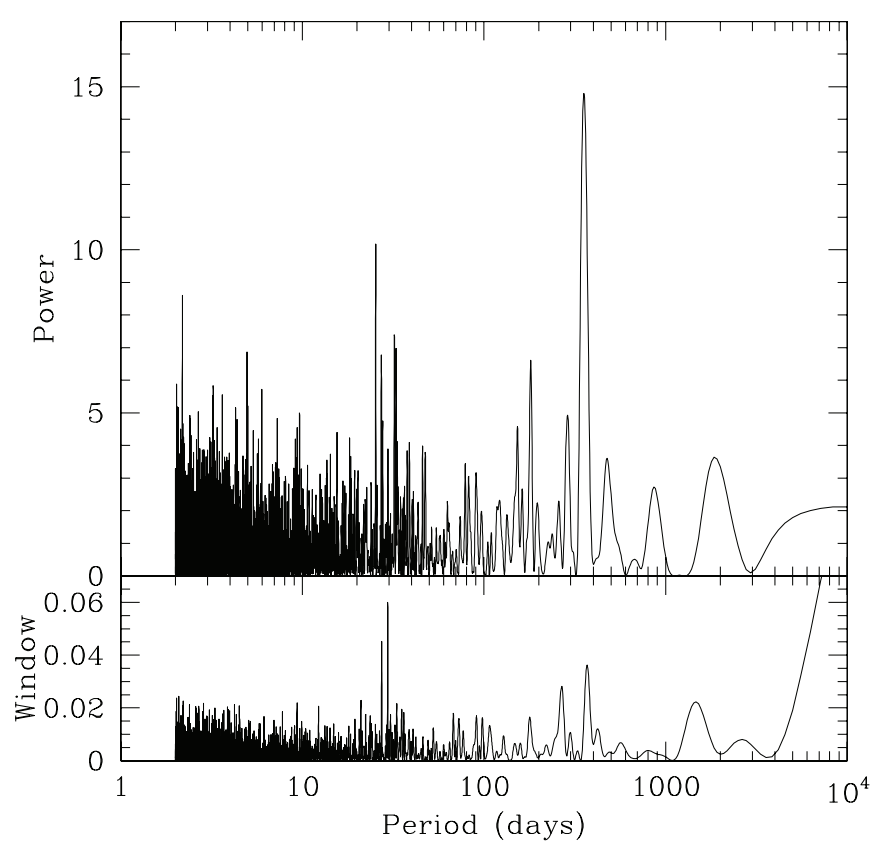

Figure 4. Periodogram of AAT residuals for HD 159868 after fitting one planet at $P=1178$ days. An additional signal is present near 355 days.

$n$-body dynamical simulations of a wide range of potential system architectures. Given the extreme uncertainty in the orbit 

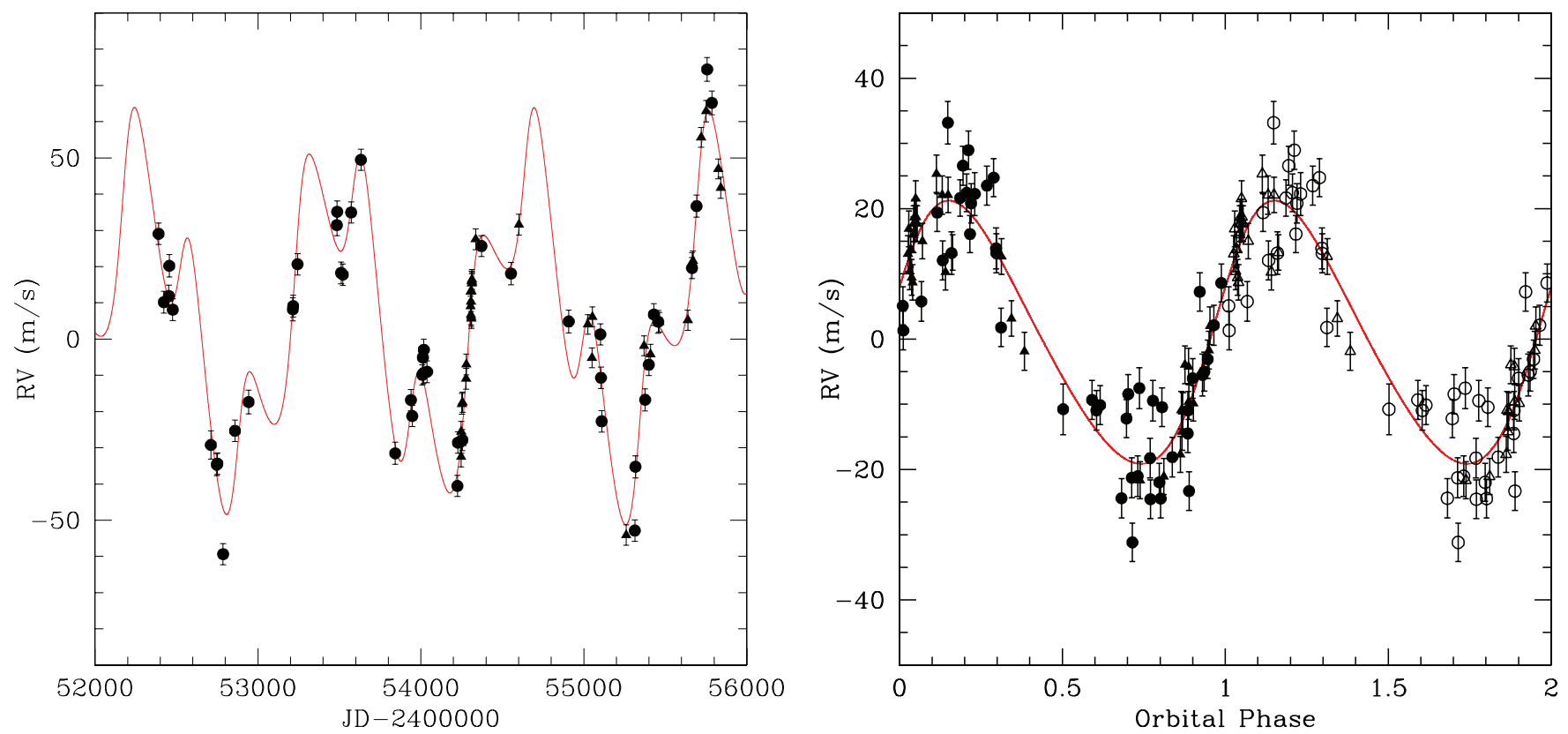

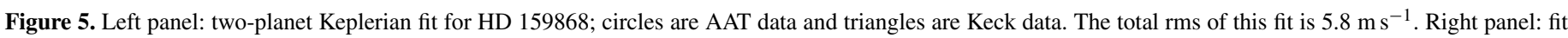
for the 355 day planet only, folded to show phase coverage. The symbols have the same meaning, and two cycles are shown for clarity.

(A color version of this figure is available in the online journal.)

Table 8

The Three-planet Solution of HD 142 Radial Velocities

\begin{tabular}{lccc}
\hline \hline Parameter & Planet b & Planet c & Residual $^{\mathrm{a}}$ \\
\hline Quit $P$ (days) & $351.1[348.3,353.8]$ & $7900[5500,22200]$ & $108.39[107.79,109.00]$ \\
$e$ & $0.15[0.00,0.34]$ & $0.18[0.00,0.72]$ & $0.12[0.00,0.56]$ \\
$K\left(\mathrm{~m} \mathrm{~s}^{-1}\right)$ & $31.6[26.1,37.1]$ & $52.6[40.0,75.9]$ & $11.6[6.1,16.5]$ \\
$\omega(\mathrm{rad})$ & $4.3[3.3,6.2]$ & $3.2[1.8,4.9]$ & $4.7[0,2 \pi]$ \\
$M_{0}(\mathrm{rad})$ & $2.6[1.4,5.1]$ & $3.0[0,2 \pi]$ & $5.3[0,2 \pi]$ \\
$m_{p} \sin i\left(M_{\text {Jup }}\right)$ & $1.21[0.88,1.54]$ & $5.5[3.7,11.6]$ & $0.30[0.15,0.45]$ \\
$a(\mathrm{AU})$ & $1.028[0.915,1.120]$ & $8.0[6.0,17.8]$ & $0.469[0.418,0.511]$ \\
\hline$\gamma\left(\mathrm{m} \mathrm{s}^{-1}\right)$ & $8.6[-5.6,74.1]$ & & \\
$\sigma\left(\mathrm{m} \mathrm{s}^{-1}\right)$ & $8.8[6.3,11.8]$ & & \\
\hline
\end{tabular}

Notes. MAP estimates of the parameters and their $99 \%$ Bayesian credibility sets.

${ }^{a}$ In the Bayesian analysis, we fit a third planet to illustrate the uncertainties in its parameters; we do not as yet claim a third planet in this system.

Table 9

The Relative Posterior Probabilities of Models $\mathcal{M}_{k}$ with $k=0, \ldots, 2$ Keplerian Signals Given the AAT Data for HD 159868

\begin{tabular}{ccc}
\hline \hline$k$ & $P\left(\mathcal{M}_{k} \mid d\right)$ & $\begin{array}{c}\mathrm{rms} \\
\left(\mathrm{m} \mathrm{s}^{-1}\right)\end{array}$ \\
\hline 0 & $4.1 \times 10^{-23}$ & 28.9 \\
1 & $1.3 \times 10^{-13}$ & 16.0 \\
2 & $\sim 1.0$ & 6.6 \\
\hline
\end{tabular}

of the outermost planet detected in the HD 142 system, we concentrated solely on the orbital stability of the 350 day planet and a possible 108 day planet. As discussed in Section 3.1, there is a residual signal after fitting two planets; the false-alarm probability of that signal is not presently low enough to justify claiming a third planet. However, if further observational data support the existence of such an object, it would be wise to understand the dynamics of the system. In this subsection, we explore the dynamical interactions between the known 350 day planet and the possible 108 day planet.
Following previous studies of exoplanetary stability (Marshall et al. 2010; Horner et al. 2011, 2012; Wittenmyer et al. 2012; Robertson et al. 2012), we used the Hybrid integrator within the $n$-body dynamics package MERCURY (Chambers 1999) to examine the stability of the planetary system as a function of its orbital architecture. Following those earlier works, we considered two-planet systems in which planet b (the most well-constrained) was placed on its nominal bestfit orbit (Table 6). The best fit for a potential third planet has $P=108.2 \pm 0.2$ days, $K=12.3 \pm 1.9 \mathrm{~m} \mathrm{~s}^{-1}, e=0.28 \pm$ 0.16 , and $\omega=271 \pm 27 \mathrm{deg}$. The initial orbital elements were then uniformly distributed across the $3 \sigma$ confidence range in $a, e$, and mean anomaly $M$. In total, we tested 35 values of $a$, 35 values of $e$, and 25 values of $M$, creating a grid of 30,625 initial system architectures. The semimajor axes for the innermost planet varied between 0.44 and 0.50 , the eccentricity was varied between 0.00 and 0.76 , and the mean anomalies were varied between 5 and $355 \mathrm{deg}$. Each of these systems was then integrated for a period of 100 million years. A body was considered to be ejected from the system if it reached a distance of $10 \mathrm{AU}$ from the central star. For each of the 30,625 potential systems, we 

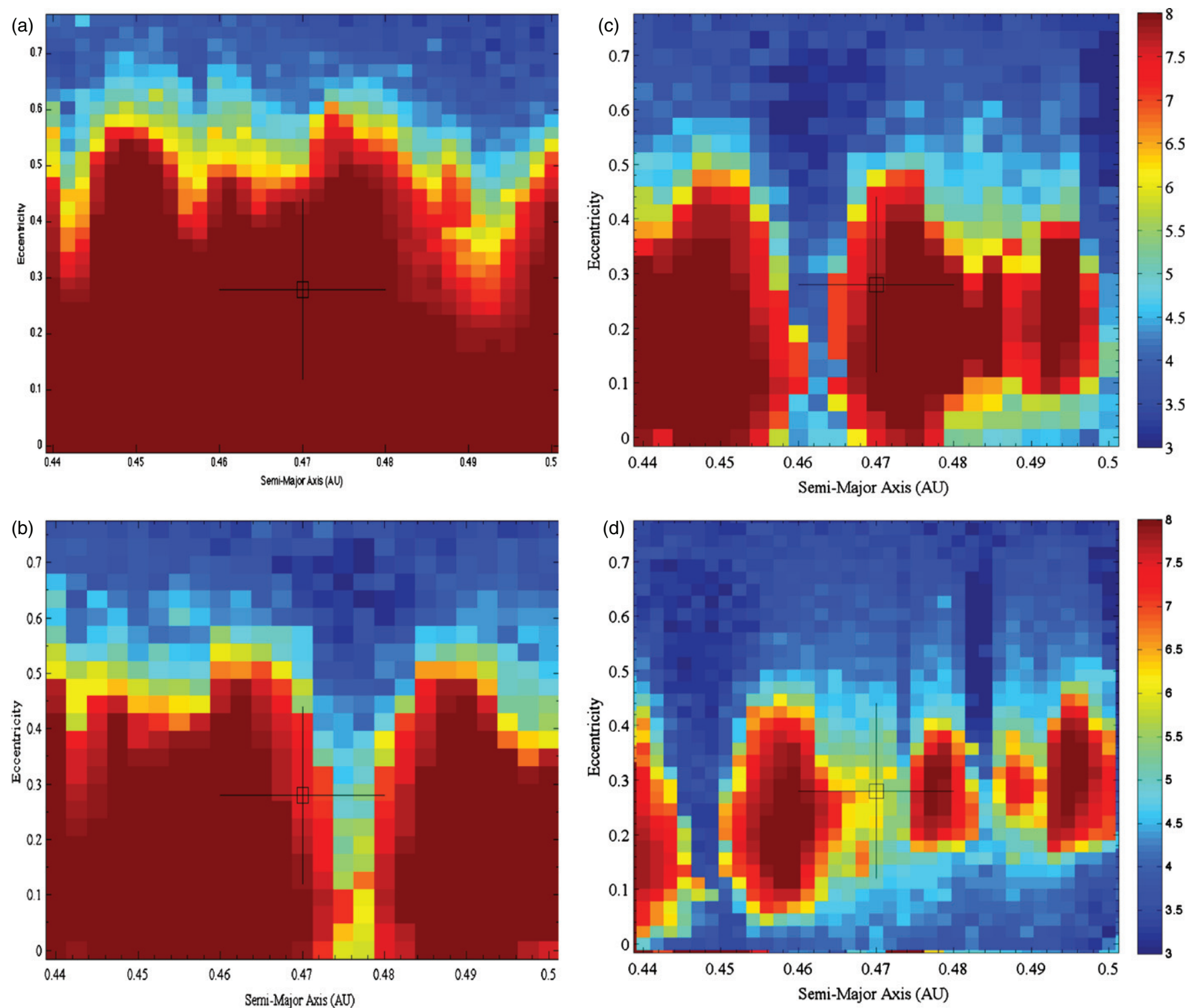

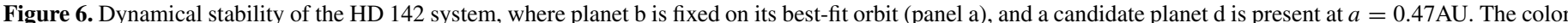

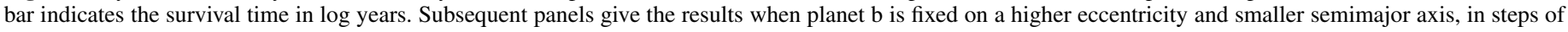

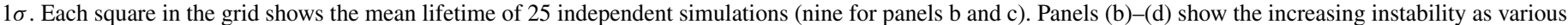
mean-motion resonances (particularly the 3:1) move with planet $b$.

(A color version of this figure is available in the online journal.)

obtained either the time at which the system fell apart (through collisions or ejections), or alternatively found that the system remained intact until the end of our simulations.

These results allow us to construct detailed dynamical maps of the system. Figure 6 shows the results: detailed dynamical maps of the HD 142 system. Each panel shows the mean lifetime of the system, as a function of semimajor axis and eccentricity, with each colored square showing the mean of the 25 individual runs carried out at that particular $a-e$ location. Panel (a) shows the results using the nominal best-fit orbit for planet $b$ : the entire region spanned by the $\pm 1 \sigma$ uncertainties on the orbit of planet $\mathrm{d}$ is dynamically stable. The only departures from stable solutions are found at relatively large orbital eccentricities for planet d, which cause the two planets to experience mutually destabilizing encounters.

Following these test integrations, we examined the most extreme case possible within the $3 \sigma$ error bounds on the orbit of planet $b$. We repeated the integrations exactly as described above, but placed planet $\mathrm{b}$ on the most eccentric orbit allowed within the $3 \sigma$ confidence interval, and at the smallest semimajor axis that interval would allow (i.e., $a=0.93 \mathrm{AU}$ and $e=0.37$ ). This was designed to give the system the greatest possible chance of instability - essentially to test it to destruction. These results are shown in panel (d) of Figure 6. In contrast to the integrations described above, the great majority of the phase space tested for this extreme scenario turns out to be unstable, although there remain several broad "islands of stability." These islands are separated by a wealth of unstable regions, primarily driven by the web of mutual mean-motion resonances between the two planets.

To better illustrate how the region of instability varies as a function of the orbit of a potential 108 day planet "d," we carried out two subsidiary suites of integrations. These again covered the full $\pm 3 \sigma$ range of $a-e$ space for planet d, but with a resolution of $25 \times 25 \times 9$ steps in $a-e-M$. First, we placed planet $\mathrm{b}$ on an orbit with eccentricity $1 \sigma$ greater than the nominal value, 
and a semimajor axis $1 \sigma$ smaller than the nominal value (i.e., $a=0.99$ AU and $e=0.27$ ). In the second suite, planet $\mathrm{b}$ was placed on an orbit $2 \sigma$ more eccentric than, and $2 \sigma$ inside, the nominal values (i.e., $a=0.96 \mathrm{AU}$ and $e=0.32$ ). These results are shown in panels (b) and (c) of Figure 6, respectively. Thus, panels (b)-(d) show the results when the orbital eccentricity and semimajor axis of planet $b$ are changed in $1 \sigma$ steps from their nominal values.

It is clear that the scenarios featured in panels (a) and (b) of Figure 6 reveal a far greater proportion of dynamically stable orbits. One obvious result from these dynamical tests is that when the eccentricity of a candidate planet $d$ increases, the stability of the system dramatically decreases. The cross at the center of each panel in Figure 6 shows the best fit and $1 \sigma$ uncertainties for a third planet. The most recent data for HD 142 now result in a higher eccentricity for a third planet $(e=0.4 \pm 0.1)$. The results of our dynamical simulations, in which higher eccentricities for a proposed planet d were less likely to remain stable, are in agreement with the analysis in Section 3.1: a 108 day planet in this system is increasingly unlikely.

\subsection{The HD 159868 System}

To test the orbital stability of the two planets discovered in the HD 159868 system, we once again performed two detailed suites of dynamical integrations of the planetary system, using the Hybrid integrator within MERCURY. In the first suite, as for the first set of runs performed for the planets in the HD 142 system, we considered scenarios in which HD159868b was placed on its nominal orbit ( $a=2.25 \mathrm{AU}, e=0.05$, etc.). We then carried out 30,625 individual simulations, through which the orbit of HD $159868 \mathrm{c}$ was varied across the full $3 \sigma$ range of allowed orbital solutions in $a, e$, and $M$ (in a $35 \times 35 \times 25$ grid, again as before). In stark contrast to the results for HD 142, which featured a significant number of unstable solutions, every single system tested for HD 159868 remained dynamically stable for the full 100 Myr of our study.

Following that first suite of integrations, we carried out a second test, in which the orbit of HD159868b was set to the most extreme allowed within the $3 \sigma$ uncertainties, with $a=2.13 \mathrm{AU}$ and $e=0.17$. Once again, we tested 30,625 unique planetary systems, with the orbit of HD $159868 \mathrm{c}$ varied across the full $3 \sigma$ range of allowed orbital solutions. These extreme runs did yield a small number of unstable solutions (162 of the 30,625 runs were destabilized by the end of the integrations), but the vast majority of systems tested survived unscathed until the end of the simulations. Every unstable solution required the initial orbit of HD 159868 c to have an eccentricity of at least 0.32 , and almost all featured initial semimajor axes of greater than 1.032 AU.

\section{DIRECT IMAGING FOR HD 142}

Since the outermost object in the HD 142 system has a very long and poorly constrained orbital period, it is prudent to check whether the observed radial-velocity variation is due to a stellar companion on a much longer-period orbit. As noted in Section 2, HD 142 is known to host a stellar companion $\left(0.56 M_{\odot}\right)$ with a projected separation of $105.1 \mathrm{AU}$. If this companion were the source of the radial-velocity signature attributed to the outermost companion, it would require the system to be almost face on (an inclination angle of $\sim 0.11 \mathrm{deg}$ for the derived $e=0.20$ and $K=55.5 \mathrm{~m} \mathrm{~s}^{-1}$ ).
Combining previous VLT-NACO observations of the HD 142 system, previously published in Eggenberger et al. (2007), and a more recent observation we made with the Near-Infrared Coronographic Imager (NICI) on the $8 \mathrm{~m}$ Gemini Telescope (Figure 7 and Table 10), we can clearly see the stellar companion moving almost directly toward the star which suggests a nearly edge-on system. The stellar companion seen from direct imaging would also have an orbital period of $>1000 \mathrm{yr}$ instead of the 6005 days (17 yr) determined from the radial-velocity data. This evidence leads us to believe that the stellar companion is not likely to be the cause of the radial-velocity variation attributed to HD 142c.

\section{DISCUSSION}

In Wittenmyer et al. (2011b), we defined a Jupiter analog as a gas-giant planet with a period $P \gtrsim 8 \mathrm{yr}$ and a small eccentricity $(e \lesssim 0.2)$. HD $142 \mathrm{c}$ has a period of 17 years, $e=0.2$, and a mass estimate consistent with a gas-giant planet. This planet thus represents a new Jupiter analog, the fourth such planet discovered by the AAPS. The three previous AAPS Jupiter analogs are HD 134987c (Jones et al. 2010), GJ 832b (Bailey et al. 2009), and HD 160691c (McCarthy et al. 2004). This discovery of a new, very long-period planet provides additional evidence that continued support for the AAPS is bearing fruit. We note that although the best-fit period for HD 142c is longer than the duration of observations, the available data cover $\sim 80 \%$ of an orbital cycle. Wittenmyer et al. (2011b) investigated the extant literature and found that the minimum orbital coverage for published planets was $70 \%$ of an orbital cycle.

The AAPS data also show hints of a third signal with a period of 108 days, evident in both the traditional periodogram analysis and the Bayesian analysis (Section 3.2). Since this signal still has a false-alarm probability of $5.1 \%$, we do not claim it to be a planet at this time. We note that while very low amplitude planets have been detected, with amplitudes comparable to the stellar radial-velocity noise (e.g., Vogt et al. 2010; Pepe et al. 2011), those detections were clearly evident in the periodograms, and the host stars were slow rotators with extremely low intrinsic jitter (unlike HD 142).

These discoveries also highlight the importance of continuing to monitor known planetary systems for signs of additional objects. In particular, both HD 142 and HD 159868 had moderately eccentric orbital solutions as well as excess scatter about the single-planet fit. A dedicated search pursuing this strategy was performed by Wittenmyer et al. (2009), who observed 22 known planetary systems for three years using the Hobby-Eberly Telescope. While that survey did not result in new planet discoveries, the new data cast doubts on the existence of the proposed planets HD 20367b (Udry et al. 2003) and HD 74156d (Bean et al. 2008; Meschiari et al. 2011).

In both the HD 142 and HD 159868 systems, further monitoring has revealed additional planets, and the best-fit eccentricities of the previously known planets have significantly decreased. Rodigas \& Hinz (2009) performers simulations which showed that moderately eccentric single-planet systems have up to a $\sim 19 \%$ probability of being zero-eccentricity two-planet systems. Figure 8 shows the distribution of eccentricity versus semimajor axis for single planets (open circles) and multiple planets (filled circles). A K-S test shows that there is only a $2.9 \%$ probability that the eccentricities of single- and multipleplanet systems are drawn from the same distribution. Previous analyses of the properties of multiple-planet systems have given the same result: that planets in multiple systems tend to have 


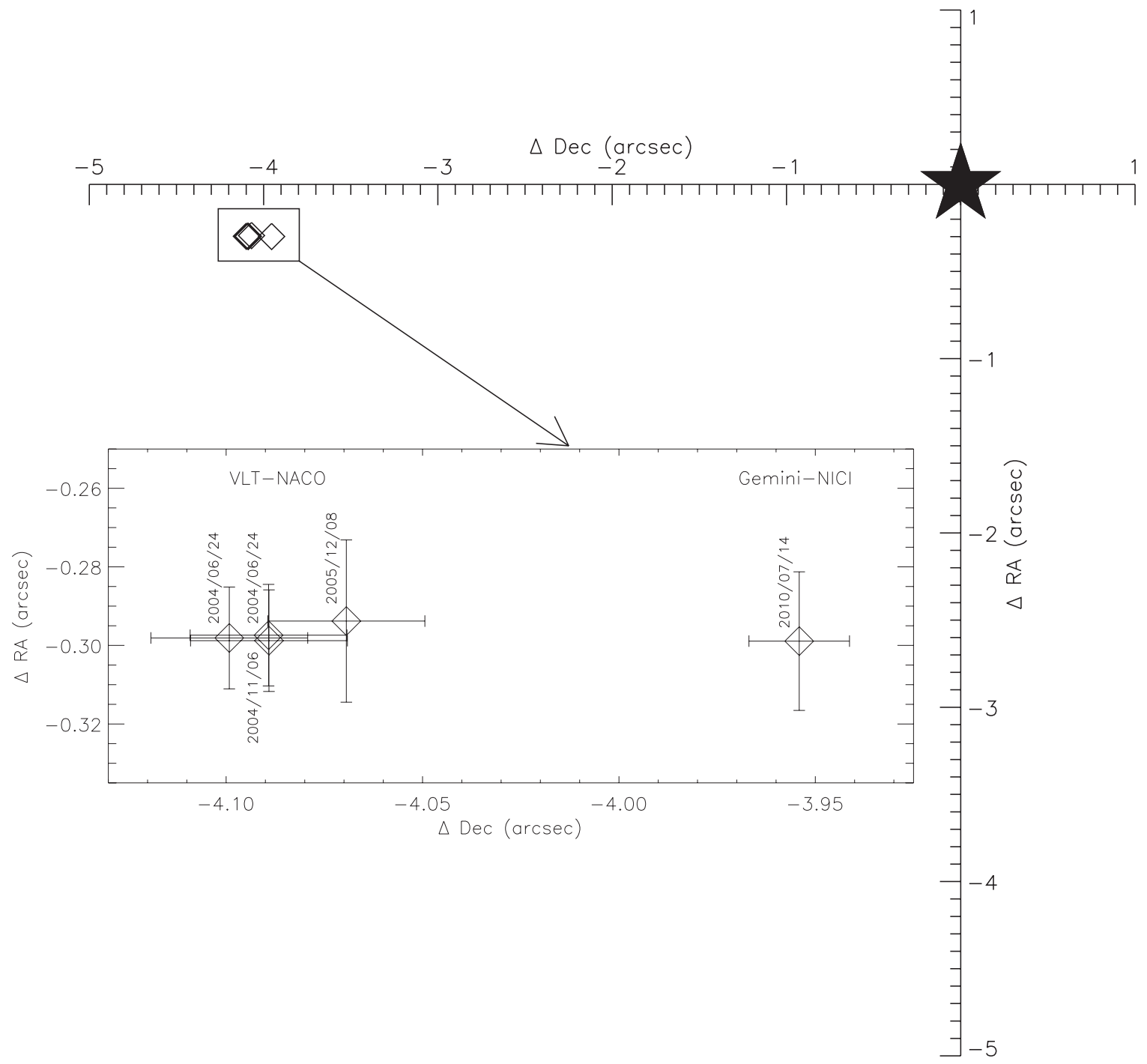

Figure 7. Observed separations between HD 142 and its stellar companion. The four data points on the left-hand side are from VLT-NACO (Eggenberger et al. 2007), and the solitary data point on the right-hand side is from Gemini-NICI observations. The date of observation is indicated next to each data point. Further details can be found in Table 10.

Table 10

Separations for HD 142 and its Stellar Companion

\begin{tabular}{lccll}
\hline \hline JD-2,400,000 & $\begin{array}{c}\text { Separation } \\
(\operatorname{arcsec})\end{array}$ & $\begin{array}{c}\text { Angle } \\
(\mathrm{deg})\end{array}$ & Instrument & Reference \\
\hline 53180.7 & $4.1 \pm 0.02$ & $184.16 \pm 0.18$ & VLT-NACO & Eggenberger et al. (2007) \\
53180.8 & $4.11 \pm 0.02$ & $184.16 \pm 0.18$ & VLT-NACO & Eggenberger et al. (2007) \\
53316 & $4.1 \pm 0.02$ & $184.18 \pm 0.18$ & VLT-NACO & Eggenberger et al. (2007) \\
53712.7 & $4.08 \pm 0.02$ & $184.13 \pm 0.29$ & VLT-NACO & Eggenberger et al. (2007) \\
55391.43 & $3.965 \pm 0.013$ & $184.47 \pm 0.26$ & Gemini-NICI & This work \\
\hline
\end{tabular}

lower eccentricities than single planets (Wittenmyer et al. 2009; Wright et al. 2009). To investigate whether this difference arises from an observational bias, we asked the following question: "Is there a minimum threshold number of observations $N$ for which single and multiple planets have the same eccentricity distribution?". We repeated the K-S tests on subsets of the known exoplanet data, including only those planets which have more than $N$ observations for a range of $N$, as shown in Table 11. If there is a bias arising from the number of observations, then for larger $N_{\text {obs }}$, there would be no significant difference in the eccentricity distributions of single- and multiple-planet systems. The K-S significance levels in Table 11 do not show any con- sistent trend with $N_{\text {obs }}$. We conclude that the difference in the eccentricity distributions of single and multiple planets is real and does not arise from the observational sampling.

Both HD 142b and HD 159868c have low-eccentricity orbits near one year about stars similar to the Sun. This raises the question of the potential habitability (e.g., Horner \& Jones 2010) of terrestrial moons which may orbit these giant planets, or Trojan companions of those planets, a topic explored in detail by Tinney et al. (2011) for HD 38283b, a gas-giant planet in a one-year orbit.

While it is somewhat speculative to discuss the potential habitability of as-yet undiscovered moons or planet-mass Trojan 


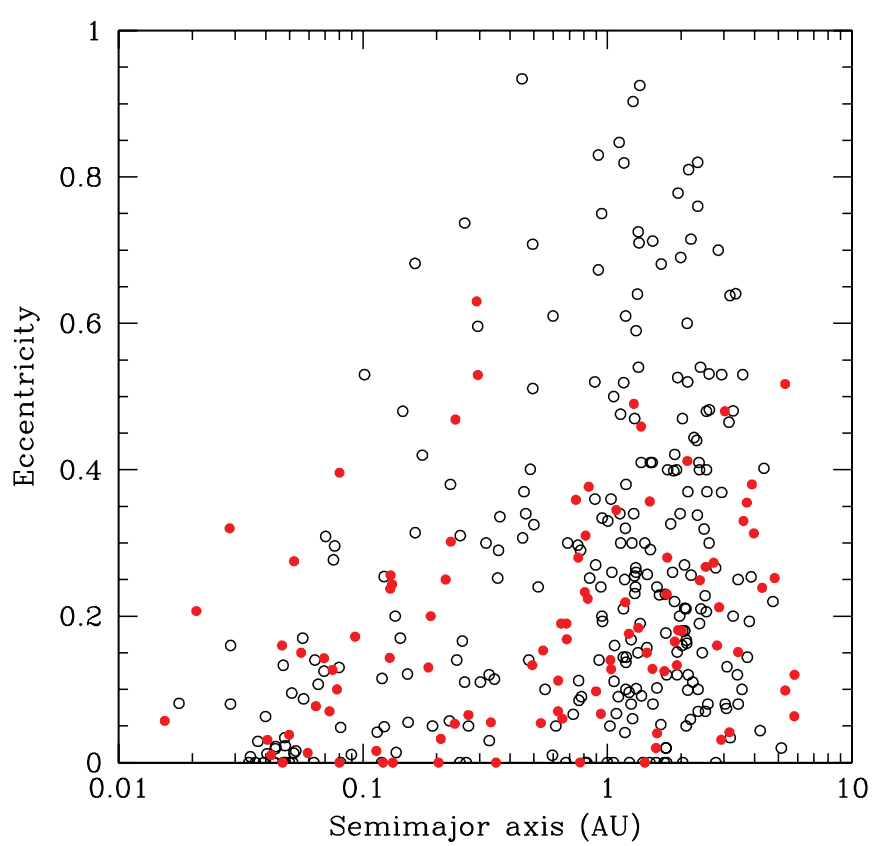

Figure 8. Distribution of eccentricity vs. semimajor axis for radial-velocity discovered planets in single systems (open circles) and multiple systems (filled circles). Planet data from the Exoplanet Orbit Database at exoplanets.org (2012 January 12). Planets in multiple systems are marginally less eccentric than single planets.

(A color version of this figure is available in the online journal.)

companions of planets such as HD $142 \mathrm{~b}$ and HD $159868 \mathrm{c}$, it is important to note that, at least for our own solar system, the capture of objects to such orbits is now considered a well-established part of planetary formation and migration. In Tinney et al. (2011), we provide an extensive review of the research into satellite formation and evolution, but it is worth reminding the reader of a few salient points. First, the combined mass of the largest satellites of the gas-giant planets in our solar system typically amounts to $2.5 \times 10^{-4}$ that of their host planet. In other words, it seems reasonable to expect that the most massive satellites of HD 142b could well be similar to, or somewhat more massive, than the Galilean satellites (since HD 142b is around 1.2 times the mass of Jupiter), while those of HD 159868c would likely be slightly less massive. As such, the regular satellites of those planets (assuming the same formation mechanism as the Galilean satellites) might well be somewhat too small to host sufficient atmosphere to allow liquid water on their surface. However, larger and more habitable satellites are clearly not beyond the bounds of possibility_particularly when one considers the possibility of the capture of massive irregular satellites (such as Neptune's moon Triton) during the course of the planet's migration (Jewitt \& Sheppard 2005; Jewitt \& Haghighipour 2007).

A more promising alternative in the search for habitable exoplanets in these systems could be the capture of objects as Trojans of the planets in question. Within the solar system, it is known that objects can be temporarily captured as Trojans for long periods of time, even in the absence of planetary migration (e.g., Horner \& Evans 2006). However, it is now widely accepted (Lykawka \& Horner 2010; Lykawka et al. 2009; Morbidelli et al. 2005) that the migration of the giant planets resulted in their capturing significant populations of Trojans. In the case of Jupiter and Neptune, those Trojan populations were captured on orbits of sufficient stability that they have survived to the current
Table 11

Eccentricity Distributions for Planets in Single and Multiple Systems

\begin{tabular}{lccc}
\hline \hline Filter & K-S Probability & $N_{\text {single }}$ & $N_{\text {multiple }}$ \\
\hline All $N_{\text {obs }}$ & 0.029 & 268 & 98 \\
$N_{\text {obs }}>40$ & 0.006 & 138 & 90 \\
$N_{\text {obs }}>50$ & 0.040 & 99 & 88 \\
$N_{\text {obs }}>60$ & 0.005 & 64 & 81 \\
$N_{\text {obs }}>70$ & 0.088 & 53 & 76 \\
$N_{\text {obs }}>80$ & 0.519 & 45 & 66 \\
$N_{\text {obs }}>90$ & 0.186 & 37 & 62 \\
$N_{\text {obs }}>100$ & 0.196 & 30 & 60 \\
$N_{\text {obs }}>110$ & 0.082 & 24 & 49 \\
$N_{\text {obs }}>120$ & 0.039 & 20 & 43 \\
$N_{\text {obs }}>130$ & 0.063 & 18 & 43 \\
$N_{\text {obs }}>140$ & 0.504 & 12 & 40 \\
\hline
\end{tabular}

Note. ${ }^{\text {a }}$ Probability that the two samples are drawn from the same distribution.

day. If either of HD 142b and HD 159868c was able to capture a sufficiently large planetary embryo as a Trojan during its inward migration to its current location, it is highly likely that such an object could remain trapped as a Trojan for the lifetime of the planetary system. If such planet-mass Trojans exist in either system, they could well represent potentially habitable worlds.

J.H. and C.G.T. gratefully acknowledge the financial support of the Australian government through ARC Grant DP0774000. R.W. is supported by a UNSW Vice-Chancellor's Fellowship. M.T. is supported by the RoPACS (Rocky Planets Around Cool Stars), a Marie Curie Initial Training Networks funded by the European Commission's Seventh Framework Programme. J.S.J. acknowledges support through Fondecyt Grant 3110004. We gratefully acknowledge the UK and Australian government support of the Anglo-Australian Telescope through their PPARC, STFC, and DIISR funding, and travel support from the Australian Astronomical Observatory and the Carnegie Institution of Washington.

We thank the ATAC for the generous allocation of telescope time which facilitated this detection. This research has made use of NASA's Astrophysics Data System (ADS), and the SIMBAD database, operated at CDS, Strasbourg, France.

\section{REFERENCES}

Anglada-Escudé, G., Arriagada, P., Vogt, S. S., et al. 2012, ApJ, 751, L16 Anglada-Escudé, G., López-Morales, M., \& Chambers, J. E. 2010, ApJ, 709, 168

Bailey, J., Butler, R. P., Tinney, C. G., et al. 2009, ApJ, 690, 743

Bean, J. L., McArthur, B. E., Benedict, G. F., \& Armstrong, A. 2008, ApJ, 672, 1202

Bond, J. C., Tinney, C. G., Butler, R. P., et al. 2006, MNRAS, 370, 163

Butler, R. P., Marcy, G. W., Williams, E., et al. 1996, PASP, 108, 500

Butler, R. P., Wright, J. T., Marcy, G. W., et al. 2006, ApJ, 646, 505

Chambers, J. E. 1999, MNRAS, 304, 793

Cochran, W. D., Endl, M., Wittenmyer, R. A., \& Bean, J. L. 2007, ApJ, 665, 1407

Diego, F., Charalambous, A., Fish, A. C., \& Walker, D. D. 1990, Proc. Soc. Photo-Opt. Instrum. Eng., 1235, 562

Eggenberger, A., Udry, S., Chauvin, G., et al. 2007, A\&A, 474, 273

Fabrycky, D. C., Ford, E. B., Steffen, J. H., et al. 2012, ApJ, 750, 114

Ford, E. B., \& Gregory, P. C. 2007, in ASP Conf. Ser. 371, Statistical Challenges in Modern Astronomy IV, ed. G. J. Babu \& E. D. Feigelson (San Francisco, CA: ASP), 189

Gonzalez, G., \& Laws, C. 2007, MNRAS, 378, 1141

Gray, R. O., Corbally, C. J., Garrison, R. F., et al. 2006, ApJ, 132, 161

Gregory, P. C., \& Fischer, D. A. 2010, MNRAS, 403, 731

Haario, H., Saksman, E., \& Tamminen, J. 2001, Bernoulli, 7, 223

Horner, J., \& Evans, N. W. 2006, MNRAS, 367, L20 
Horner, J., \& Jones, B. W. 2010, Int. J. Astrobiol., 9, 273

Horner, J., Marshall, J. P., Wittenmyer, R. A., \& Tinney, C. G. 2011, MNRAS, 416, L11

Horner, J., Wittenmyer, R. A., Marshall, J. P., Tinney, C. G., \& Butters, O. W. 2012, in Proc. 11th Australian Space Sci. Conf., Canberra, 26-29 September, 2011, ed. W. Short \& I. Cairns (Canberra: National Space Society of Australia), 13

Houk, N. 1978, Michigan Catalogue of Two-dimensional Spectral Types for the HD Stars (Ann Arbor, MI: Dept. of Astronomy, University of Michigan: Distributed by University Microfilms International)

Isaacson, H., \& Fischer, D. 2010, ApJ, 725, 875

Jefferys, W. H., Fitzpatrick, M. J., \& McArthur, B. E. 1987, Celest. Mech., 41, 39

Jenkins, J. S., Jones, H. R. A., Tinney, C. G., et al. 2006, MNRAS, 372, 163

Jewitt, D., \& Haghighipour, N. 2007, ARA\&A, 45, 261

Jewitt, D., \& Shepard, S. 2005, Space Sci. Rev., 116, 441

Johnson, J. A., Aller, K. M., Howard, A. W., \& Crepp, J. R. 2010, PASP, 122, 905

Jones, H. R. A., Butler, R. P., Tinney, C. G., et al. 2010, MNRAS, 403, 1703

Kjeldsen, H., \& Bedding, T. R. 1995, A\&A, 293, 87

Kürster, M., Schmitt, J. H. M. M., Cutispoto, G., \& Dennerl, K. 1997, A\&A, 320,831

Lang, K. R. 1980, Astrophysical Formulae. A Compendium for the Physicist and Astrophysicist (2nd enlarged and revised edn.; Berlin: Springer)

Lykawka, P. S., \& Horner, J. 2010, MNRAS, 405, 1375

Lykawka, P. S., Horner, J., Jones, B. W., \& Mukai, T. 2009, MNRAS, 398, 1715

Malyuto, V., \& Shvelidze, T. 2011, Balt. Astron., 20, 91

Marshall, J., Horner, J., \& Carter, A. 2010, Int. J. Astrobiol., 9, 259

Mayor, M., Marmier, M., Lovis, C., et al. 2011, A\&A, submitted (arXiv:1109.2497)

McCarthy, C., Butler, R. P., Tinney, C. G., et al. 2004, ApJ, 617, 575

Meschiari, S., Laughlin, G., Vogt, S. S., et al. 2011, ApJ, 727, 117

Morbidelli, A., Levison, H. F., Tsiganis, K., \& Gomes, R. 2005, Nature, 435, 462

O’Toole, S. J., Butler, R. P., Tinney, C. G., et al. 2007, ApJ, 660, 1636

O’Toole, S. J., Jones, H. R. A., Tinney, C. G., et al. 2009, ApJ, 701, 1732
Pepe, F., Lovis, C., Ségransan, D., et al. 2011, A\&A, 534, A58

Perryman, M. A. C., Lindegren, L., Kovalevsky, J., et al. 1997, A\&A, 323, L49 Poveda, A., Herrera, M. A., Allen, C., Cordero, G., \& Lavalley, C. 1994, RevMexAA, 28, 43

Raghavan, D., Henry, T. J., Mason, B. D., et al. 2006, ApJ, 646, 523

Ramírez, I., Allende Prieto, C., \& Lambert, D. L. 2007, A\&A, 465, 271

Randich, S., Gratton, R., Pallavicini, R., Pasquini, L., \& Carretta, E. 1999, A\&A, 348,487

Rivera, E. J., Lissauer, J. J., Butler, R. P., et al. 2005, ApJ, 634, 625

Robertson, P., Endl, M., Cochran, W. D., et al. 2012, ApJ, 749, 39

Rodigas, T. J., \& Hinz, P. M. 2009, ApJ, 702, 716

Sousa, S. G., Santos, N. C., Mayor, M., et al. 2008, A\&A, 487, 373

Takeda, G., Ford, E. B., Sills, A., et al. 2007, ApJS, 168, 297

Tinney, C. G., Butler, R. P., Marcy, G. W., et al. 2002, ApJ, 571, 528

Tinney, C. G., Wittenmyer, R. A., Butler, R. P., et al. 2011, ApJ, 732, 31

Tuomi, M. 2011, A\&A, 528, L5

Tuomi, M., \& Kotiranta, S. 2009, A\&A, 496, L13

Tuomi, M., Pinfield, D., \& Jones, H. R. A. 2011, A\&A, 532, A116

Udry, S., Mayor, M., Benz, W., et al. 2006, A\&A, 447, 361

Udry, S., Mayor, M., \& Queloz, D. 2003, in ASP Conf. Ser. 294, Scientific Frontiers in Research on Extrasolar Planets, ed. D. Deming \& S. Seager (San Francisco, CA: ASP), 17

Valenti, J. A., Butler, R. P., \& Marcy, G. W. 1995, PASP, 107, 966

Valenti, J. A., \& Fischer, D. A. 2005, ApJS, 159, 141

van Belle, G. T., \& von Braun, K. 2009, ApJ, 694, 1085

van Leeuwen, F. 2007, A\&A, 474, 653

Vogt, S. S., Allen, S. L., Bigelow, B. C., et al. 1994, Proc. SPIE, 2198, 362

Vogt, S. S., Wittenmyer, R. A., Butler, R. P., et al. 2010, ApJ, 708, 1366

Wittenmyer, R. A., Endl, M., Cochran, W. D., Levison, H. F., \& Henry, G. W. 2009, ApJS, 182, 97

Wittenmyer, R. A., Endl, M., Wang, L., et al. 2011a, ApJ, 743, 184

Wittenmyer, R. A., Horner, J., Marshall, J. P., Butters, O. W., \& Tinney, C. G. 2012, MNRAS, 419, 3258

Wittenmyer, R. A., Tinney, C. G., O'Toole, S. J., et al. 2011b, ApJ, 727, 102

Wright, J. T. 2005, PASP, 117, 657

Wright, J. T., Upadhyay, S., Marcy, G. W., et al. 2009, ApJ, 693, 1084 